\title{
Eotaxin-1 and MCP-1 serve as circulating indicators in response to power frequency electromagnetic field exposure in mice
}

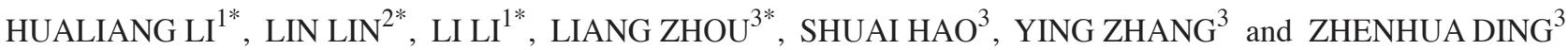 \\ ${ }^{1}$ Institute of Environmental Protection, Guangdong Power Grid, Guangzhou, Guangdong 510080; \\ ${ }^{2}$ Department of Obstetrics, The Sixth Affiliated Hospital of Sun Yat-sen University, Guangzhou, Guangdong 510655; \\ ${ }^{3}$ Department of Radiation Medicine, Guangdong Provincial Key Laboratory of Tropical Disease Research, \\ School of Public Health, Southern Medical University, Guangzhou, Guangdong 510515, P.R. China
}

Received October 24, 2017; Accepted June 12, 2018

DOI: $10.3892 / \mathrm{mmr} .2018 .9237$

\begin{abstract}
The increasing public concern regarding the potential health risks of exposure to electromagnetic fields (EMFs) has led to intensive research in this area. However, it remains unclear whether potential pro-oncogenic effects may be caused by power frequency EMF (PFEMF) exposure. To address the associated risk factors, the present study exposed 4-week old Balb/c mice to 0, 0.1, 0.5 and $2.5 \mathrm{mT}$ of constant $50 \mathrm{~Hz}$ Helmholtz coil-type PFEMF for 90 days to explore the circulating chemokine indicators that may be associated with inflammation or cancer. No measurable weight difference existed between the control and PFEMF-exposure groups; however, the Luminex assay clearly demonstrated differentially responsive profiles of circulating chemokines upon PFEMF treatment. Monocyte chemoattractant protein (MCP)-3, macrophage inflammatory protein (MIP)-1 $\alpha$, MIP-1 $\beta$ and MIP-2 levels in serum were not significantly altered by PFEMF during the 3-month exposure period; however, the circulating levels of other chemokines including IP-10, GRO $\alpha$, RANTES, EOTAXIN-1 and MCP-1 exhibited significant changes upon treatment. Among the responsive chemokines, EOTAXIN-1 and MCP-1 were significantly increased by $0.5 \mathrm{mT}$ of PFEMF treatment, which may support their use as indicators of PFEMF exposure. This novel finding highlights the potential pro-inflammatory nature of power frequency, which may shed light on the mechanisms underlying PFEMF-induced diseases, including cancer.
\end{abstract}

Correspondence to: Professor Zhenhua Ding, Department of Radiation Medicine, Guangdong Provincial Key Laboratory of Tropical Disease Research, School of Public Health, Southern Medical University, 1838 Northern Guangzhou Avenue, Guangzhou, Guangdong 510515, P.R. China

E-mail:dingzh@smu.edu.cn

*Contributed equally

Key words: power frequency electromagnetic fields, chemokine, Eotaxin-1, monocyte chemoattractant protein-1

\section{Introduction}

Power frequency electromagnetic field (PFEMF) of 50-60-Hz, a type of non-ionizing radiation generated by household appliances, transmission lines, and transformers, belongs to the category of extreme-low frequency electromagnetic fields (ELF-EMF) (3-300 Hz). Although PFEMF differs from radiofrequency, which was suggested to be possibly carcinogenic to humans by the World Health Organization (WHO) in 2014, concerns regarding the potential effects of PFEMF on human health have been widely debated in the past decades. This debate has been stimulated by epidemiological studies indicating a relationship between ELF-EMF and various types of cancer (1-4). Additionally, arguments supporting both no significant (5) and significant $(6,7)$ health risks have led to increased apprehension owing to the lack of sufficient mechanistic understanding regarding the biological effects of PFEMF (8).

The only endpoint studied in sufficient detail with respect to the relationship between PFEMF and cancer concerns childhood leukemia, which is the most frequent childhood malignancy and peaks in the age group of 2- to 5-year-olds. Most cases consist of acute lymphocytic leukemia or acute myeloid leukemia; conversely, chronic leukemias are rare in children. Evidence also suggests that childhood leukemia is tightly associated with PFEMF exposure during pregnancy or early life and risk estimates reach statistical significance at exposure levels of 0.3-0.4 $\mu \mathrm{T}$ (9-12). Notably, childhood cancers aside from leukemia such as brain and nervous system tumors have not been studied in sufficient detail to draw conclusions regarding the existence and magnitude of the potential risks associated with PFEMF (13). Furthermore, to date, no mechanism by which ELF-EMFs or other types of radiofrequency radiation might cause cancer has been identified.

Chemokines belong to a family of small cytokines, 8-10 kDa in mass, that are secreted by cells. Chemokines function as chemotactic cytokines to induce the directed migration of leukocytes after interacting with their corresponding receptors, a process termed chemotaxis (2). Based on their structure, chemokines can be classified into four highly conserved groups: $\mathrm{CC}, \mathrm{CXC}, \mathrm{C}$, and $\mathrm{CX}_{3} \mathrm{C}$. More than 50 chemokines and at least 18 chemokine receptors have been identified (14). In addition, 
many chemokines are pro-inflammatory and can be induced during an immune response to recruit cells of the immune system to a site of infection. Strong evidences have indicated that complex networks of chemokines and their receptors play diverse roles and influence the development of primary cancers and metastases (14-20). In particular, the levels of a CC chemokine, CCL18, have been shown to be correlated with the incidence of childhood leukemia, and thus this chemokine has been proposed to act as a diagnostic marker $(19,21)$. In adult leukemia, higher expression levels of the chemokine receptor CCR4 have also been found to indicate poor prognosis (15). CCL2-triggered chemokine cascade in macrophages promotes metastatic seeding of breast cancer cells thereby amplifying the extant pathology (17). Furthermore, chemokines are not only associated with the occurrence of cancer but also determine the metastatic capabilities of primary cancer together with integrin molecules $(18,20)$.

The purpose of this study was to investigate whether exposure to PFEMF might dysregulate the circulating chemokine levels in serum, which we considered might serve as a prerequisite for establishing a relationship between the occurrence of malignancy and PFEMF exposure. We divided mice into groups and treated them with different doses of a magnetic field ( $0.1 \mathrm{mT}$ to represent public exposure, $0.5 \mathrm{mT}$ for occupational exposure, and $2.5 \mathrm{mT}$ for unusually strong exposure) for $8 \mathrm{~h}$ every day. The serum of the treated animals was collected at different time points $(0,1,10,30$, and 90 days $)$ and subjected to chemokine assays using the Luminex technique (22) to determine the presence of chemokine induction by PFEMF. Evidence supporting the potential pro-inflammatory nature of power frequency may shed light on the mechanisms of PMEMF-induced diseases, including cancer.

\section{Materials and methods}

Animals. The present study was approved by the Institutional Animal Care and Use Committee (IACUC) of Southern Medical University (Approval code: L2016103; 13 September 2016). They are in accordance with the guidelines of the Asian Federation of Laboratory Animal Science Associations (AFLAS) and the National Regulations for the Administration of Affairs Concerning Experimental Animals (8 January 2011). All animals were purchased from the experimental animal center of Southern Medical University and treated in accordance with standard guidelines for the care and use of laboratory animals. Male Balb/c mice weighing $17.05 \pm 0.2 \mathrm{~g}$ at the time of the experiments were housed at $25^{\circ} \mathrm{C}$ and $50-60 \%$ relative humidity in cages under a 12-12 h light/dark cycle, with free access to food and water. The mice were divided into groups and exposed to different dosages of magnetic field $(0.1,0.5$, and $2.5 \mathrm{mT}$ with $50 \mathrm{~Hz}, 8 \mathrm{~h} /$ day) for different numbers of consecutive days $(0,1,10,30$, and 90 days). Out of 100 animals in each group, 20 were randomly chosen and weighed every other day.

Exposure system. The exposure system was assembled by Lioncel (Shanghai, China) and was composed of a TSGC2J-60KVA contact type voltage regulator (Goodyi, Shanghai, China) and a model MFA-201C magnetic antenna (Lioncel). The transformer was used to provide electric current to the Helmholtz-type coils for generating the magnetic field, and the homogenous region of which was $0.4 \times 0.4 \times 0.4$ (m) with harmonics below $1.0 \%$. The PFEMF was measured inside the cage and kept constant, independent of the position. As 'on-off' switching operations induce high-frequency transient EMFs that may lead to biological effects, we always switched on the system, waited for at least 1 min to ensure the absence of transients, and then placed the cages in the coils with mechanical assistance. The whole equipment assembly and cage placement was depicted in Fig. 1A.

Exposure procedure. The exposed groups $(\mathrm{n}=100$ for each dosage of $0.1,0.5$, and $2.5 \mathrm{mT}$ ) and equal number mice of control group were housed in standardized cages $(10$ mice per cage); all cages were placed in the same room. In order to simulate the working and living environmental conditions, the PFEMF exposure group was exposed to EMF of $50 \mathrm{~Hz}$ at 0.1 , 0.5 , or $2.5 \mathrm{mT}$ (rms), as determined by a gaussmeter (PMM 8053B; NARDA Safety Test Solutions, Italy), for $8 \mathrm{~h}$ per day for $0,1,10,30$, and 90 consecutive days. Exposed animals were compared with sham-exposed controls derived from the same source and simultaneously handled and assessed in the same manner, except for the presence of the EMFs. The animals were weighed every other day using an electronic balance.

Serum collection. At the planned time points, blood was harvested from the retro-orbital plexus after the mice were anesthetized using diethyl ether. The serum samples were collected as described previously (23). The blood samples were incubated at $4^{\circ} \mathrm{C}$ for $3-4 \mathrm{~h}$ to allow clotting and then centrifuged at $2,500 \mathrm{~g}$ at $4^{\circ} \mathrm{C}$ for $10 \mathrm{~min}$. Isolated serum (approximately $300 \mu \mathrm{l}$ ) was preserved at $-80^{\circ} \mathrm{C}$ for further analysis. For each dosage-time point, at least 20 serum samples were collected. The mice were euthanized by cervical dislocation to prevent suffering after blood sampling. The Southern Medical University Animal Care and Use Committee approved all procedures involving the mice.

Chemokine assay. The concentrations of chemokines were detected using a ProcartaPlex ${ }^{\circledR}$ Multiplex Immunoassay Chemokine Panel 1 [(eBioscience; Thermo Fisher Scientific, Inc., Waltham, MA, USA), 9 plex, including EOTAXIN-1 (CCL11), GRO $\alpha$ (CXCL1), IP-10 (CXCL10), MCP-1 (CCL2), MCP-3 (CCL7), MIP-1 $\alpha$ (CCL3), MIP-1 $\beta$ (CCL4), MIP-2 (CXCL2), and RANTES (CCL5)] via the Luminex technique. For each dosage-time point, 4 serum samples were used for the Luminex assay, and their chemokine concentrations were averaged as the final concentration of that dosage-time point. This assay was performed following the manufacturer's instructions. The evaluation of the inter-assay coefficients of variation is described in Table I.

Enzyme-linked immunosorbent assay (ELISA). The concentrations of EOTAXIN-1 and MCP-1 were measured using mouse CCL2/MCP-1 and CCL11/Eotaxin ELISA kits (MultiSciences, Shanghai, China), respectively, to verify the levels of the two chemokines in serum. The tests were performed according to the manufacturer's instructions for each kit. Density values for unknown samples were assessed using the standard curve for each analyte to calculate actual values in $\mathrm{pg} / \mathrm{ml}$. The minimum 
Table I. Coefficients of variation for low and high value controls of the measured variables.

\begin{tabular}{|c|c|c|c|c|c|c|c|c|}
\hline \multirow[b]{2}{*}{ Variables } & \multirow[b]{2}{*}{ Units } & \multicolumn{3}{|c|}{ Control I (low values) } & \multicolumn{3}{|c|}{ Control II (high values) } & \multirow{2}{*}{$\begin{array}{c}\text { Inter-assay } \\
\mathrm{CV}(\mathrm{n}=4 ; \%)\end{array}$} \\
\hline & & Range & Mean & $\mathrm{CV}(\%)$ & Range & Mean & $\mathrm{CV}(\%)$ & \\
\hline МCP-1 & $\mathrm{pg} / \mathrm{ml}$ & $53.18-89.20$ & 68.62 & 28.06 & $95.33-114.90$ & 107.72 & 6.27 & 17.17 \\
\hline MCP-3 & $\mathrm{pg} / \mathrm{ml}$ & $128.04-215.75$ & 174.31 & 19.63 & $63.76-102.75$ & 83.85 & 16.95 & 18.29 \\
\hline MIP-1 $1 \alpha$ & $\mathrm{pg} / \mathrm{ml}$ & $2.55-3.58$ & 3.11 & 16.71 & $4.40-6.15$ & 5.25 & 10.16 & 13.43 \\
\hline MIP-1 $\beta$ & $\mathrm{pg} / \mathrm{ml}$ & $2.34-7.27$ & 4.91 & 28.64 & $7.90-11.46$ & 9.58 & 14.72 & 21.68 \\
\hline MIP-2 & $\mathrm{pg} / \mathrm{ml}$ & $14.14-30.00$ & 20.25 & 29.26 & $10.19-13.67$ & 11.91 & 9.35 & 19.31 \\
\hline EOTAXIN-1 & $\mathrm{pg} / \mathrm{ml}$ & $87.26-176.76$ & 127.06 & 27.25 & $388.54-551.62$ & 473.70 & 11.12 & 19.18 \\
\hline IP-10 & $\mathrm{pg} / \mathrm{ml}$ & $57.91-124.51$ & 84.58 & 29.67 & 62.33-134.47 & 97.72 & 25.34 & 27.51 \\
\hline GRO- $\alpha$ & $\mathrm{pg} / \mathrm{ml}$ & $16.63-29.24$ & 22.14 & 30.97 & $12.29-35.16$ & 25.43 & 27.11 & 29.04 \\
\hline RANTES & $\mathrm{pg} / \mathrm{ml}$ & 7.04-14.31 & 10.90 & 33.51 & $19.39-36.03$ & 26.02 & 20.64 & 27.08 \\
\hline
\end{tabular}

$\mathrm{MCP}$, monocyte chemoattractant protein; MIP, macrophage inflammatory protein; CV, coefficients of variation.
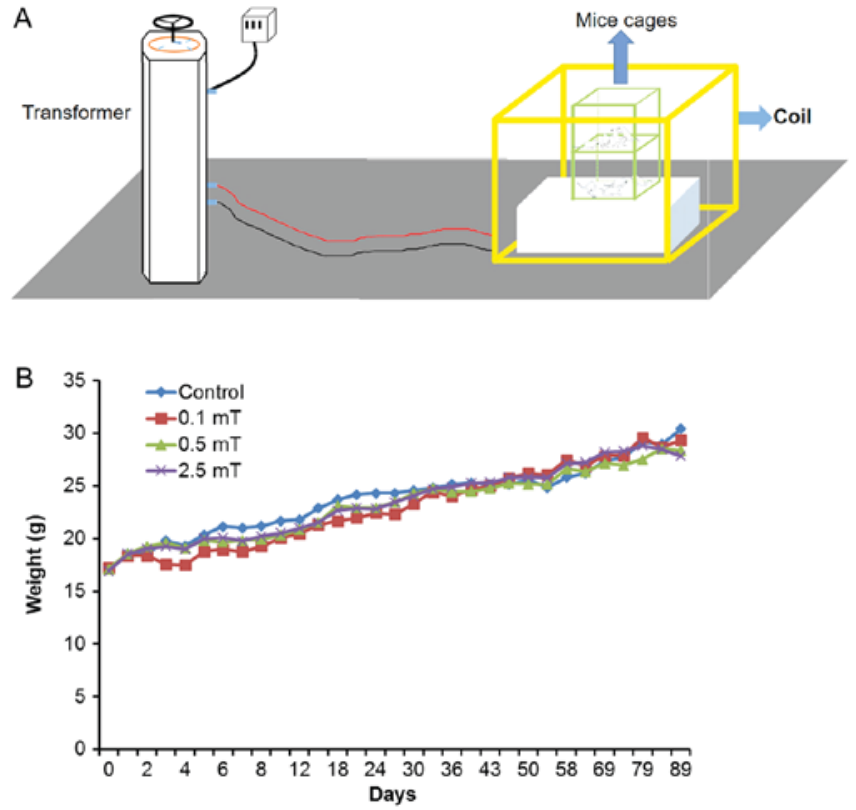

Figure 1. PFEMF exposure system and comparing the temporal profiles of weight among the control and treatment groups. (A) Schematic of the PFEMF exposure system, which was assembled with a transformer and coil. (B) The body weight curves were generated by randomly selecting 20 mice from each of the 4 groups and weighing them at each indicated time point. PFEMF, power frequency electromagnetic fields.

detectable dose of CCL2 was $20.54 \mathrm{pg} / \mathrm{ml}$ and that of CCL11 was $0.73 \mathrm{pg} / \mathrm{ml}$.

Statistical analysis. IBM SPSS statistics v.20 software (IBM Corp., Armonk, NY, USA) was used for data analysis. All observations were tested for normality using the Kolmogorov-Smirnov test and for homogeneity of variance using Levene's test. Factorial analysis of variance followed by Dunnett's post hoc test was used for comparisons between experimental and controls groups for mouse weight and chemokine levels (all statistical tests performed were two-sided). The results are expressed as the mean \pm standard error (SE). $\mathrm{P}<0.05$ was considered to indicate a statistically significant difference.

\section{Results}

PFEMF exposure does not lead to significant change of body weight. In order to simulate the working and living environmental conditions, the animals were exposed to EMF of $50 \mathrm{~Hz}$ at $0.1,0.5$, or $2.5 \mathrm{mT}$, for $8 \mathrm{~h}$ per day for $0,1,10$, 30 , and 90 consecutive days. For the control group, the initial and final body weight averages were $17.1 \pm 0.6$ and $30.4 \pm 0.2 \mathrm{~g}$, respectively; for the $0.1 \mathrm{mT}$ group, the respective values were $17.2 \pm 0.4$ and $29.4 \pm 0.6 \mathrm{~g}$; for the $0.5 \mathrm{mT}$ group, the respective values were $17.1 \pm 0.5$ and $29.4 \pm 0.4 \mathrm{~g}$; and for the $2.5 \mathrm{mT}$ group, the respective values were $16.9 \pm 0.4$ and $27.8 \pm 0.2 \mathrm{~g}$. $\mathrm{N}=20$ for each group. Although the treated groups show slight decreases compared with the control group overall, no significant difference was detected. From these results, we concluded that PFEMF exposure did not cause a significant alteration in body weight (Fig. 1B).

PFEMF exposure induces specific circulating chemokines in mice. To investigate the relationship between alteration of chemokine concentration in serum and exposure to PFEMF, we determined the circulating chemokine concentration in serum using the Luminex technique after $0,1,10,30$, and 90 days of PFEMF exposure. The levels of some of the assayed chemokines, e.g., MCP-3, MIP-1 $\alpha$, MIP-1 $\beta$, and MIP-2, in the serum were not significantly influenced by PFEMF exposure (Table II; Fig. 2A). Conversely, statistical analysis showed that different dosages of PFEMF treatment may have caused significant variations in the levels of several chemokines including IP-10, GRO $\alpha$, RANTES, EOTAXIN-1, and MCP-1. Among them, IP-10, GRO $\alpha$, and RANTES showed a response to only one of three doses, whereas levels of EOTAXIN-1 and MCP-1 were significantly increased by PFEMF exposure of $0.5 \mathrm{mT}$ alone (Table II; Fig. 2A). Beyond the initial induction response during the first 10 days of exposure, the medium and high dose (0.5 and $2.5 \mathrm{mT})$ led to the upregulation of MCP-1 and this trend was maintained for three months (Day 30 to Day 90) (Table II; Fig. 2B). For EOTAXIN-1, all of the three tested doses caused a drastic induction of EOTAXIN-1 levels in the serum at the beginning of treatment, whereas 
Table II. Levels of chemokines in response to PFEMF exposure were determined by the Luminex assay.

\begin{tabular}{|c|c|c|c|c|c|c|c|c|c|}
\hline \multirow[b]{2}{*}{ Chemokine } & \multirow{2}{*}{$\begin{array}{l}\text { Dose } \\
(\mathrm{mT})\end{array}$} & \multirow[b]{2}{*}{ Day 0} & \multirow[b]{2}{*}{ Day 1} & \multirow[b]{2}{*}{ Day 10} & \multirow[b]{2}{*}{ Day 30} & \multirow[b]{2}{*}{ Day 90} & \multicolumn{3}{|c|}{$\begin{array}{c}\text { Variable } \\
\text { comparisons (P-values) }\end{array}$} \\
\hline & & & & & & & Dose & Time & Dose ${ }^{*}$ Time \\
\hline \multirow[t]{4}{*}{ MCP-1 } & 0 & $93.91 \pm 3.23$ & $102.98 \pm 4.30$ & $104.98 \pm 4.07$ & $96.91 \pm 3.56$ & $107.43 \pm 6.99$ & - & - & - \\
\hline & 0.1 & $93.91 \pm 3.23$ & $111.98 \pm 0.97$ & $104.3 \pm 6.08$ & $97.23 \pm 4.52$ & $103.59 \pm 3.37$ & $\mathrm{P}=0.046^{\mathrm{a}}$ & $\mathrm{P}<0.001^{\mathrm{b}}$ & $\mathrm{P}=0.510$ \\
\hline & 0.5 & $93.91 \pm 3.23$ & $111.29 \pm 5.60$ & $111.64 \pm 1.74$ & $111.47 \pm 2.07$ & $129.02 \pm 11.14$ & & & \\
\hline & 2.5 & $93.91 \pm 3.23$ & $119.2 \pm 7.88$ & $100.55 \pm 12.65$ & $103.8 \pm 3.31$ & $111.51 \pm 3.43$ & & & \\
\hline \multirow[t]{4}{*}{ MCP-3 } & 0 & $54.45 \pm 4.46$ & $76.70 \pm 9.78$ & $101.57 \pm 9.14$ & $83.57 \pm 5.57$ & $130.42 \pm 9.75$ & - & - & - \\
\hline & 0.1 & $54.45 \pm 4.46$ & $116.53 \pm 16.28$ & $80.00 \pm 6.38$ & $82.24 \pm 10.99$ & $90.70 \pm 3.05$ & $\mathrm{P}=0.108$ & $\mathrm{P}<0.001^{\mathrm{b}}$ & $\mathrm{P}=0.001^{\mathrm{b}}$ \\
\hline & 0.5 & $54.45 \pm 4.46$ & $117.32 \pm 14.17$ & $85.28 \pm 5.85$ & $88.59 \pm 8.37$ & $165.26 \pm 31.98$ & & & \\
\hline & 2.5 & $54.45 \pm 4.46$ & $102.51 \pm 13.84$ & $105.16 \pm 14.55$ & $105.30 \pm 10.57$ & $68.19 \pm 8.43$ & & & \\
\hline \multirow[t]{4}{*}{ MIP-1 $\alpha$} & 0 & $5.12 \pm 0.50$ & $9.32 \pm 3.84$ & $5.37 \pm 0.26$ & $4.65 \pm 0.28$ & $5.00 \pm 0.50$ & - & - & - \\
\hline & 0.1 & $5.12 \pm 0.50$ & $8.13 \pm 0.59$ & $4.65 \pm 0.31$ & $4.69 \pm 0.22$ & $4.91 \pm 0.24$ & $\mathrm{P}=0.919$ & $\mathrm{P}=0.017^{\mathrm{a}}$ & $\mathrm{P}=0.577$ \\
\hline & 0.5 & $5.12 \pm 0.50$ & $6.03 \pm 0.25$ & $5.06 \pm 0.25$ & $10.03 \pm 4.18$ & $5.35 \pm 0.78$ & & & \\
\hline & 2.5 & $5.12 \pm 0.50$ & $7.82 \pm 0.81$ & $4.54 \pm 0.69$ & $5.84 \pm 0.88$ & $4.30 \pm 0.32$ & & & \\
\hline \multirow[t]{4}{*}{ MIP-1 $\beta$} & 0 & $9.26 \pm 0.99$ & $10.32 \pm 1.16$ & $9.66 \pm 0.45$ & $6.57 \pm 0.48$ & $8.15 \pm 0.74$ & - & - & - \\
\hline & 0.1 & $9.26 \pm 0.99$ & $12.74 \pm 0.92$ & $7.98 \pm 0.79$ & $7.54 \pm 0.36$ & $7.40 \pm 0.32$ & $\mathrm{P}=0.501$ & $\mathrm{P}<0.001^{\mathrm{b}}$ & $\mathrm{P}=0.294$ \\
\hline & 0.5 & $9.26 \pm 0.99$ & $10.96 \pm 0.59$ & $9.01 \pm 0.49$ & $9.49 \pm 0.16$ & $9.00 \pm 1.01$ & & & \\
\hline & 2.5 & $9.26 \pm 0.99$ & $10.27 \pm 0.40$ & $7.82 \pm 0.73$ & $8.12 \pm 0.45$ & $7.84 \pm 0.36$ & & & \\
\hline \multirow[t]{4}{*}{ MIP-2 } & 0 & $11.74 \pm 0.43$ & $12.06 \pm 0.74$ & $12.58 \pm 0.66$ & $10.76 \pm 0.80$ & $12.97 \pm 0.74$ & - & - & - \\
\hline & 0.1 & $11.74 \pm 0.43$ & $13.53 \pm 0.74$ & $11.09 \pm 0.57$ & $11.70 \pm 0.31$ & $13.08 \pm 1.17$ & $\mathrm{P}=0.702$ & $\mathrm{P}<0.001^{\mathrm{b}}$ & $\mathrm{P}=0.115$ \\
\hline & 0.5 & $11.74 \pm 0.43$ & $12.68 \pm 0.36$ & $13.60 \pm 0.57$ & $11.44 \pm 0.41$ & $13.51 \pm 0.62$ & & & \\
\hline & 2.5 & $11.74 \pm 0.43$ & $14.02 \pm 0.68$ & $12.79 \pm 0.84$ & $10.88 \pm 0.68$ & $11.36 \pm 0.64$ & & & \\
\hline \multirow[t]{4}{*}{ EOTAXI-1 } & 0 & $301.59 \pm 27.19$ & $458.30 \pm 37.01$ & $535.10 \pm 41.28$ & $440.42 \pm 15.25$ & $564.53 \pm 46.36$ & - & - & - \\
\hline & 0.1 & $301.59 \pm 27.19$ & $835.48 \pm 63.25$ & $500.92 \pm 40.91$ & $520.94 \pm 15.48$ & $580.08 \pm 23.52$ & $\mathrm{P}<0.001^{\mathrm{b}}$ & $\mathrm{P}<0.001^{\mathrm{b}}$ & $\mathrm{P}<0.001^{\mathrm{b}}$ \\
\hline & 0.5 & $301.59 \pm 27.19$ & $795.80 \pm 37.84$ & $706.19 \pm 36.55$ & $781.46 \pm 51.89$ & $866.86 \pm 88.29$ & & & \\
\hline & 2.5 & $301.59 \pm 27.19$ & $816.31 \pm 52.48$ & $519.00 \pm 109.82$ & $556.09 \pm 58.65$ & $607.92 \pm 56.25$ & & & \\
\hline \multirow[t]{4}{*}{ IP-10 } & 0 & $80.09 \pm 6.34$ & $90.36 \pm 14.09$ & $106.35 \pm 9.59$ & $55.36 \pm 2.27$ & $128.58 \pm 29.29$ & - & - & - \\
\hline & 0.1 & $80.09 \pm 6.34$ & $107.41 \pm 13.47$ & $81.67 \pm 9.15$ & $60.79 \pm 7.52$ & $53.00 \pm 1.13$ & $\mathrm{P}=0.734$ & $\mathrm{P}=0.277$ & $\mathrm{P}=0.232$ \\
\hline & 0.5 & $80.09 \pm 6.34$ & $92.66 \pm 4.41$ & $72.81 \pm 3.88$ & $67.44 \pm 8.46$ & $125.02 \pm 65.92$ & & & \\
\hline & 2.5 & $80.09 \pm 6.34$ & $107.26 \pm 1.98$ & $96.44 \pm 17.87$ & $95.49 \pm 33.54$ & $54.84 \pm 8.92$ & & & \\
\hline \multirow[t]{4}{*}{ GRO- $\alpha$} & 0 & $29.37 \pm 3.56$ & $24.95 \pm 5.66$ & $33.52 \pm 5.50$ & $15.08 \pm 2.67$ & $25.10 \pm 6.71$ & - & - & - \\
\hline & 0.1 & $29.37 \pm 3.56$ & $25.78 \pm 7.17$ & $21.55 \pm 3.12$ & $19.52 \pm 2.76$ & $23.84 \pm 9.06$ & $\mathrm{P}=0.056$ & $\mathrm{P}=0.229$ & $\mathrm{P}=0.070$ \\
\hline & 0.5 & $29.37 \pm 3.56$ & $28.84 \pm 2.81$ & $31.12 \pm 1.99$ & $32.41 \pm 5.56$ & $66.54 \pm 12.84$ & & & \\
\hline & 2.5 & $29.37 \pm 3.56$ & $35.11 \pm 3.64$ & $29.46 \pm 6.88$ & $27.16 \pm 9.83$ & $20.61 \pm 2.37$ & & & \\
\hline \multirow[t]{4}{*}{ RANTES } & 0 & $26.59 \pm 1.52$ & $27.75 \pm 3.23$ & $27.23 \pm 1.14$ & $26.43 \pm 1.64$ & $37.01 \pm 11.16$ & - & - & - \\
\hline & 0.1 & $26.59 \pm 1.52$ & $35.44 \pm 2.64$ & $23.80 \pm 1.11$ & $21.79 \pm 0.93$ & $22.36 \pm 0.43$ & $\mathrm{P}=0.338$ & $\mathrm{P}=0.159$ & $\mathrm{P}=0.300$ \\
\hline & 0.5 & $26.59 \pm 1.52$ & $28.44 \pm 2.56$ & $25.45 \pm 1.02$ & $23.93 \pm 1.31$ & $30.97 \pm 11.14$ & & & \\
\hline & 2.5 & $26.59 \pm 1.52$ & $37.58 \pm 3.76$ & $23.56 \pm 2.62$ & $20.48 \pm 3.64$ & $17.45 \pm 1.09$ & & & \\
\hline
\end{tabular}

Data are plotted as the mean \pm standard error. ${ }^{\mathrm{a}} \mathrm{P}<0.05$ and ${ }^{\mathrm{b}} \mathrm{P}<0.001$, all 3 experimental groups vs. control in regard to the dose groups, time, and dose and time combined. PFEMF, power frequency electromagnetic fields; MCP, monocyte chemoattractant protein; MIP, macrophage inflammatory protein.

EOTAXIN-1 levels returned to normal at day 10 for both the 0.1 and $2.5 \mathrm{mT}$ treatments. However, $0.5 \mathrm{mT}$ exposure led to consistent upregulation of EOTAXIN-1 following the initial induction response (Table II; Fig. 2C).

ELISA verification indicates that MCP-1 and EXTAXIN-1 may be used as indicators of PFEMF exposure. To confirm the results from the Luminex assay, we subjected the same samples to ELISA detection. Treatments using the three PFEMF doses $(0.1,0.5$, and $2.5 \mathrm{mT})$ led to a significant increase in the concentration of EOTAXIN-1. Generally, the serum concentrations of MCP-1 and EOTAXIN-1 from animals treated with $0.5 \mathrm{mT}$ showed the highest increase at all time points relative to the control group. For the $2.5 \mathrm{mT}$ treatment, only EOTAXIN-1 showed a statistically significant difference compared with the control group (Table III; Fig. 3). 
A

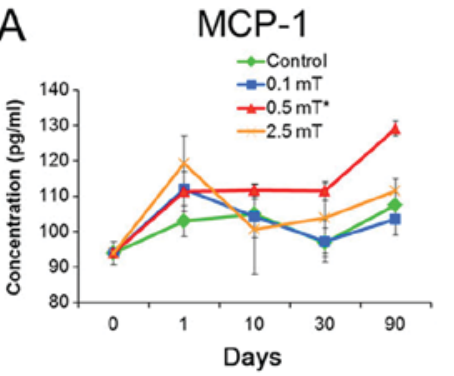

MIP-1 $\beta$

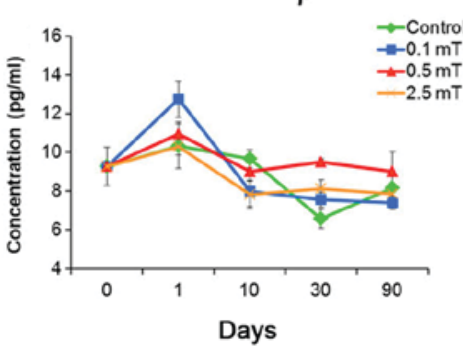

IP-10

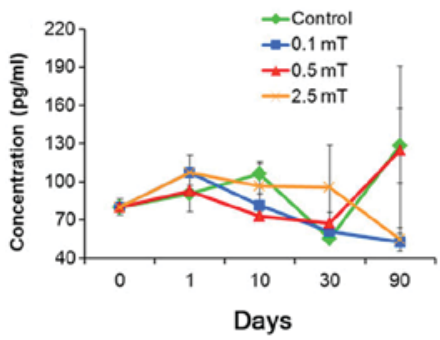

B

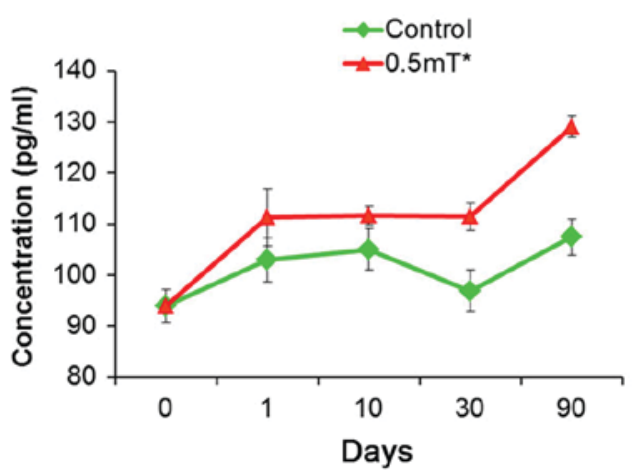

MCP-3

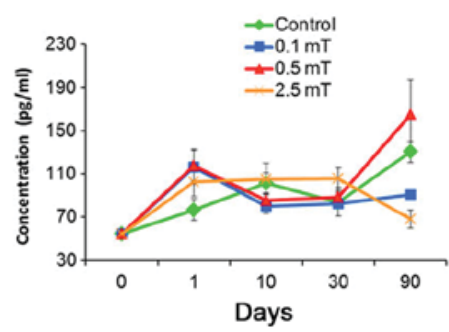

MIP-2

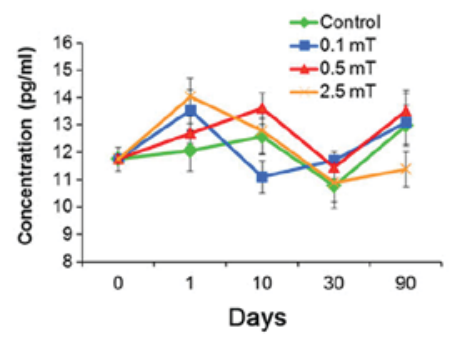

$\mathrm{GRO} \alpha$

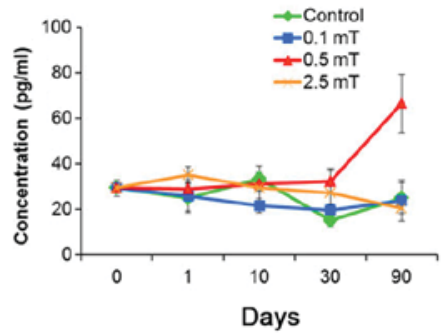

C
MIP-1 $\alpha$

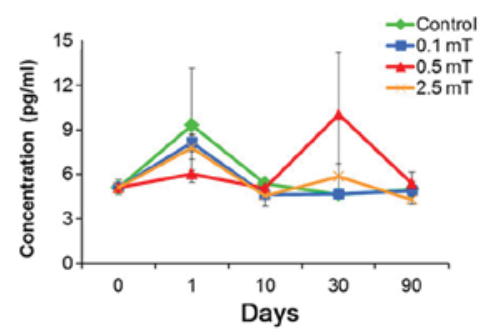

EOTAXIN-1

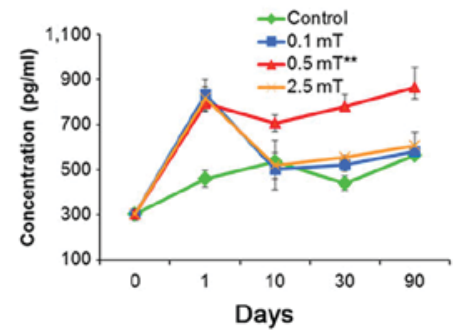

RANTES

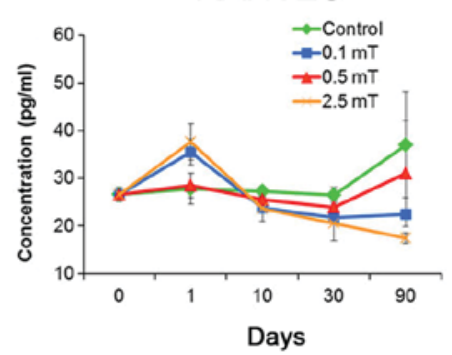

\section{EOTAXIN-1}

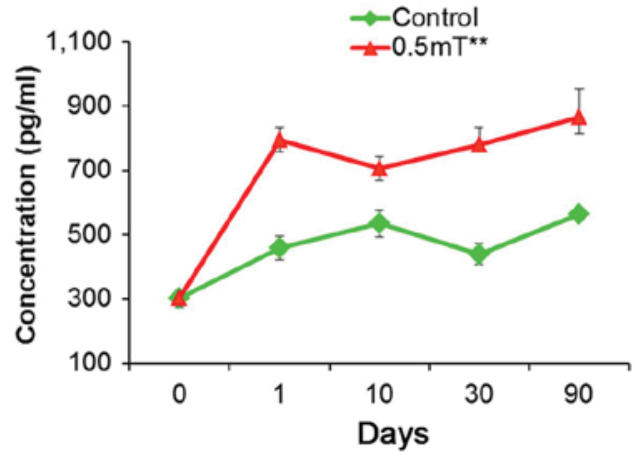

Figure 2. Dosage-time concentration profiles of chemokines as determined by the Luminex assay upon power frequency magnetic field exposure. (A) The concentrations of 9 chemokines were determined using the Luminex technique. The dosage-time concentration profile, determined by Luminex, of (B) MCP-1 and (C) EOTAXIN-1 in the $0.5 \mathrm{mT}$ group was also compared with the control group alone. Data are plotted as the mean \pm standard error ( $\mathrm{n}=4$ per assessment). ${ }^{*} \mathrm{P}<0.05$ and ${ }^{* *} \mathrm{P}<0.01$ vs. control (at all time points combined). MCP, monocyte chemoattractant protein; MIP, macrophage inflammatory protein.

Notably, the variation curves of EOTAXIN-1 concentration as detected by ELISA were approximately the same as those determined using Luminex technology.

\section{Discussion}

In this study, to investigate the circulating chemokine indicators and eatablish a solid base for solving the increasing public concern regarding the potential health risks from PFEMF, mice were exposed to different doses $(0,0.1,0.5$, and $2.5 \mathrm{mT})$ of PFEMF for as long as 90 days. Among the 9 chemokines, EOTAXIN-1 and MCP-1 were constantly induced by $0.5 \mathrm{mT}$ of PFEMF treatment and may serve as dose-specific PFEMF exposure indicators.

Our previous study utilized computer-based neurobehavioral evaluation methods to investigate the neurobehavioral influences on employees working with electric power systems and who thus were exposed to relatively strong PFEMFs (24). This investigation demonstrated that no significant variation could be found among different age or seniority groups 
Table III. Serum levels of Eotaxin-1 and MCP-1 in response to PFEMF exposure were determined by ELISA.

Variable comparison (P-value)

Dose

Chemokine (mT)

Day 0

Day 1

Day 10

Day 30

Day 90

Dose

\begin{tabular}{|c|c|c|c|c|c|c|c|}
\hline \multirow[t]{4}{*}{ MCP-1 } & 0 & $801.91 \pm 76.76$ & $857.85 \pm 121.86$ & $818.89 \pm 109.78643 .38 \pm 39.74$ & $427.40 \pm 24.61$ & - & - \\
\hline & 0.1 & & $802.37 \pm 73.86$ & $899.27 \pm 35.51 \quad 715.48 \pm 58.79$ & $436.86 \pm 55.14$ & $\mathrm{P}=0.009^{\mathrm{b}} \mathrm{P}<0.001^{\mathrm{c}}$ & $\mathrm{P}=0.332$ \\
\hline & 0.5 & & $992.97 \pm 19.94$ & $1,044.79 \pm 103.01824 .73 \pm 95.11$ & $613.14 \pm 48.74$ & & \\
\hline & 2.5 & & $791.99 \pm 31.84$ & $1,136.17 \pm 155.02696 .64 \pm 118.31$ & $461.29 \pm 33.72$ & & \\
\hline \multirow[t]{4}{*}{ EOTAXIN-1 } & 0 & $131.39 \pm 10.38$ & $190.76 \pm 19.67$ & $258.10 \pm 20.82 \quad 226.52 \pm 13.65$ & $271.67 \pm 21.75$ & - & - \\
\hline & 0.1 & & $355.55 \pm 59.87$ & $201.31 \pm 12.35 \quad 260.01 \pm 27.04$ & $257.04 \pm 20.73$ & $\mathrm{P}=0.001^{\mathrm{c}} \mathrm{P}<0.001^{\mathrm{c}}$ & $\mathrm{P}=0.014^{\mathrm{a}}$ \\
\hline & 0.5 & & $348.72 \pm 24.51$ & $295.96 \pm 41.65$ & $365.52 \pm 21.95$ & & \\
\hline & 2.5 & & $412.75 \pm 42.38$ & $264.84 \pm 13.42 \quad 283.29 \pm 12.38$ & $336.10 \pm 17.53$ & & \\
\hline
\end{tabular}

Data are plotted as the mean \pm standard error. ${ }^{\mathrm{a}} \mathrm{P}<0.05,{ }^{\mathrm{b}} \mathrm{P}<0.01$ and ${ }^{\mathrm{c}} \mathrm{P} \leq 0.001$, all 3 experimental groups vs. control in regard to the dose groups, time, and dose and time combined. PFEMF, power frequency electromagnetic fields; MCP, monocyte chemoattractant protein.

A

MCP-1

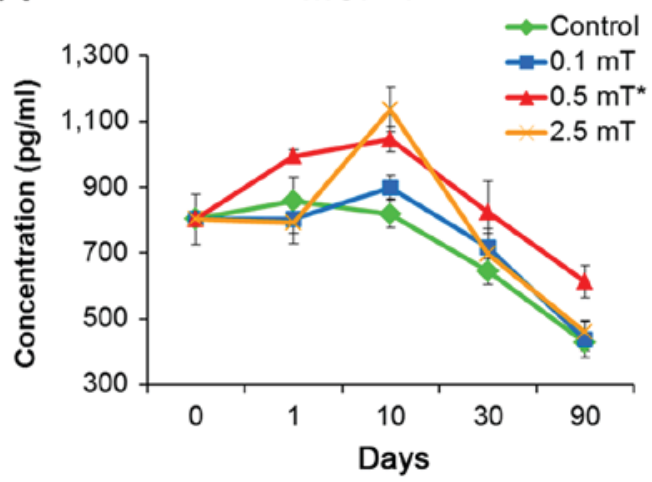

C

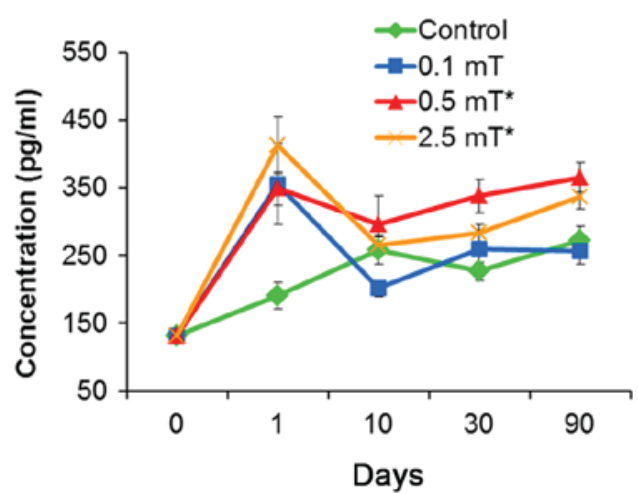

B MCP-1

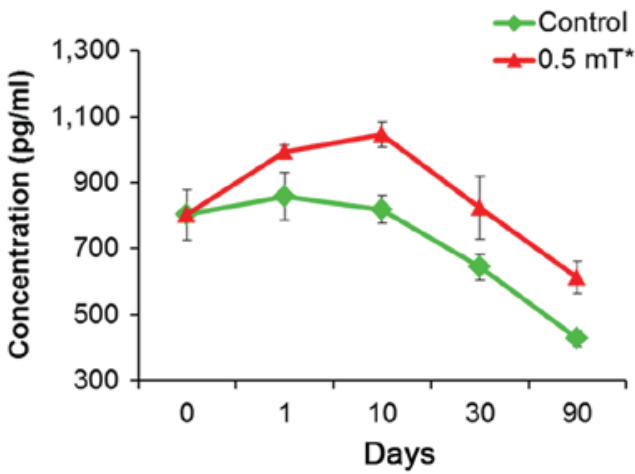

EOTAXIN-1

D

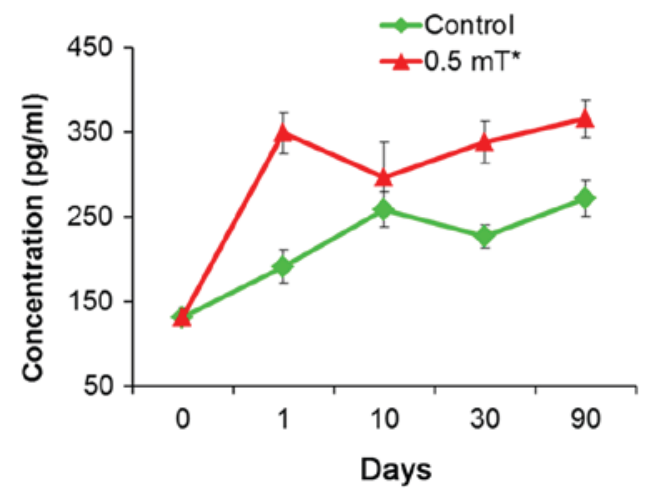

Figure 3. Dosage-time concentration profiles of EOTAXIN-1 and MCP-1 as determined by ELISA upon power frequency magnetic field exposure. The concentration of (A) MCP-1 or (C) EOTAXIN-1 was measured using a mouse CCL2/MCP-1 or CCL11/Eotaxin ELISA kit, respectively. The dosage-time concentration profile of (B) MCP-1 or (D) EOTAXIN-1 of the $0.5 \mathrm{mT}$ group, as determined by ELISA, were compared with the control group. Data are plotted as the mean \pm standard error $(n=4)$. ${ }^{*} \mathrm{P}<0.05$ and ${ }^{* *} \mathrm{P}<0.01$ vs. control (at all time points combined). MCP-1, monocyte chemoattractant protein 1 ; CCL, C-C motif chemokine ligand.

after short- or long-term exposure in response to strong PFEMF (24). Considering the large differences within many variables observed in that study, we simplified the study in the present study by using a homogeneous mouse model to examine the influences of the electromagnetic field generated by PFEMF. Furthermore, to facilitate the reflection of normal human life circumstances and occupational environments, we selected the magnetic flux density of $0.1 \mathrm{mT}$ to represent public exposure and $0.5 \mathrm{mT}$ for occupational exposure, which are the levels adopted by the Chinese National Standards for 
the electricity industry and are also more stringent than those used in the current criteria of the International Commission on Non-Ionizing Radiation Protection (ICNIRP). In addition, the relatively higher dose of $2.5 \mathrm{mT}$ was also included to represent unusually strong PFEMF exposure. These values allowed us to explore the variations in chemokine levels associated with cancer or inflammation and to begin to elucidate the indicators of exposure and associated disease to address current concerns regarding the effects of PFEMF in surrounding environments. In the present study, we did not observe any measurable difference in body weight between the control and treatment groups, which is in line with the findings of our previous study (24) and indicates that PFEMF is unlikely to cause apparent phenotypic differences in the exposed population.

Chemokines are secreted by cells in response to various types of in vivo and in vitro stimuli and have been shown to play important roles in carcinogenesis $(14,15,19,20,25,26)$. Specifically, chronic inflammation, a well-known risk factor for cancer, is tightly associated with the dysregulation of chemokines, which can directly regulate tumor cell growth and migration. We found that certain chemokines exhibited differential responses to PFEMF exposure. Levels of some chemokines in the serum were not significantly changed following 3-month exposure, such as MCP-3, MIP-1 $\alpha$, MIP-1 $\beta$, and MIP-2, whereas levels of others including IP-10, GRO $\alpha$, RANTES, EOTAXIN-1, and MCP-1 demonstrated significant changes upon treatment. Among them, we observed that IP-10, GRO $\alpha$, and RANTES were responsive to only one of the three dosages, whereas EOTAXIN-1 and MCP-1 were significantly increased by at least two doses of PFEMF treatment. Furthermore, we determined that MCP-1 was constantly induced following $0.5 \mathrm{mT}$ exposure but not by $2.5 \mathrm{mT}$, although both doses also caused significant changes in EOTAXIN-1 levels.

MCP-1 (CCL2) can regulate the migration and infiltration of many leukocytes including $\mathrm{T}$ cells, natural killer cells, and monocytes, and has been shown to be induced and involved in various diseases, especially cancers $(27,28)$. MCP-1 is a mediator of acute and chronic inflammation and can be secreted from the microenvironment to promote tumorigenesis and cancer progression $(29,30)$. MCP-1 has also been demonstrated to be induced upon overnight exposure to $1 \mathrm{mT}-50 \mathrm{~Hz}$ ELF-EMF treatment in human peripheral adherent mononuclear cells (31). In contrast, results from other studies using cultured cell lines including HaCaT, SH-SY5Y, THP-1, and K562 cells suggested that the expression of MCP-1 was repressed or unaffected after similar treatments, possibly as a result of inhibition of the NF- $\kappa \mathrm{B}$ pathway $(32,33)$. However, the anti-inflammatory potency of EMFs detected in cell lines in vitro may not reflect the in vivo situation. The induction of MCP-1 by PFEMF in an animal model as illustrated by our work highlights the critical importance of using animal models for health-related investigations in this area.

EOTAXIN-1 (CCL11) is one of the CC family chemokines and is characterized by a pair of adjacent cysteine residues. EOTAXIN-1 serves as a potent chemoattractant for eosinophils and plays critical pro-inflammatory roles in phenomena such as eosinophilia, which is a prominent feature of several allergic conditions. EOTAXIN-1 promotes cell proliferation, invasion, and angiogenesis in ovarian cancer, prostate cancer, and Hodgkin's lymphoma, as demonstrated by the activation of the CCR3-ERK pathway or the upregulation of MMP-3 expression or $\mathrm{CCR}^{+}$endothelial cells (24-36). In particular, as EOTAXIN-1 is highly induced upon carcinogenesis, the serum levels of EOTAXIN-1 can be used as a biomarker for an enhanced risk of gastric cancer and ovarian cancer and as a prognostic factor for the prediction of relapse-free survival $(34,37)$. However, few reports to date have studied the relationship between EOTAXIN and PFEMF (38). The induction of EOTAXIN-1 by PFEMF in an animal model as shown by our work therefore constitutes a novel finding that suggests a pro-inflammatory effect of PFEMF exposure.

The non-dose-dependent responses of chemokine is out of our expectation. This phenomenon may reflect the body has an unknown mechanism to respond to different electromagnetic field strength which is worth to further explore. We also noticed that some of the cytokines are fluctuated during PFEMF treatment and do not follow a linear augmenting pattern (Table II). To our knowledge and understanding, our finding of MCP-1 and EOTAXIN-1 as stable dose-specific indicators is valuable and lays a solid foundation for establishing a systemic diagnostic procedure of PFEMF exposure. The induction of the two circulating chemokines, EOTAXIN-1 and MCP-1, upon PFEMF treatment strongly supports their potential application as indicators for exposure to PFEMF, which may be especially useful for convenient public health monitoring and thus contribute to the final goal of establishing a healthy living environment. Also, our study asked an open question, why MCP-1 and EOTAXIN-1 is responsive to $0.5 \mathrm{mT}$ ELF-EMF exposure, but not the other doses? Any further mechanistic study for this question may have profound impact in clinical diagnosis. We note, however, that the exact concentrations of EOTAXIN-1 and MCP-1 as determined by the Luminex and ELISA assays differed somewhat, which may be due to the influences of differential sensitivity or detection limitations of the two techniques. Further, owing to the limited energy conveyed by power frequency, it is difficult to directly observe the potential pro-oncogenic role of PFEMF exposure in normal mice. Future studies using spontaneous tumor-forming animal models generated by knocking out tumor suppressors such as p53 will likely be required for validation of the potential pro-oncogenic role and mechanism of PFEMFs.

In the present study, we identified differentially responsive circulating chemokines following exposure to PFEMF using a mouse model. The novel finding of the induction of EOTAXIN-1 and MCP-1 during this process supports that these two chemokines could be used as circulating indicators for PFEMF exposure and highlights the potential pro-inflammatory nature of PFEMF, which deepens the current understanding of the biological effects of PFEMF exposure.

\section{Acknowledgements}

Not applicable.

\section{Funding}

The present study was financially supported by The Science and Technology Program of China Southern Power Grid Company (grant nos. K-GD2014-0618 and K-GD2011-415). 


\section{Availability of data and materials}

The datasets used and/or analyzed during the present study are available from the corresponding author on reasonable request.

\section{Authors' contributions}

HL, LZ and ZD conceived and designed the experiments. HL, LnL, LL, LZ, SH and YZ performed the experiments, and HL, LZ and SH analyzed the data. HL, LnL, LZ and ZD wrote the manuscript.

\section{Ethics approval and consent to participate}

The present study was approved by the Institutional Animal Care and Use Committee of Southern Medical University (Guangdong, China; approval code, L2016103; 13th September 2016).

\section{Patient consent for publication}

Not applicable.

\section{Competing interests}

The authors declare that they have no competing interests.

\section{References}

1. Kheifets LI, Afifi AA, Buffler PA and Zhang ZW: Occupational electric and magnetic field exposure and brain cancer: A meta-analysis. J Occup Environ Med 37: 1327-1341, 1995.

2. Ross CL and Harrison BS: An introduction to electromagnetic field therapy and immune function: A brief history and current status. J Sci Appl: BioMed 3: 18-29, 2015.

3. Singh S and Kapoor N: Health implications of electromagnetic fields, mechanisms of action, and research needs. Adv Biol 2014: Article ID 198609, 2014 (http://dx.doi.org/10.1155/2014/198609).

4. Wertheimer $\mathrm{N}$ and Leeper EM: Electrical wiring configurations and childhood cancer. Am J Epidemiol 109: 273-284, 1979.

5. Ikeda K, Shinmura Y, Mizoe H, Yoshizawa H, Yoshida A, Kanao S, Sumitani H, Hasebe S, Motomura T, Yamakawa T, et al: No effects of extremely low frequency magnetic fields found on cytotoxic activities and cytokine production of human peripheral blood mononuclear cells in vitro. Bioelectromagnetics 24: 21-31, 2003

6. Håkansson N, Gustavsson P, Sastre A and Floderus B Occupational exposure to extremely low frequency magnetic fields and mortality from cardiovascular disease. Am J Epidemiol 158: 534-542, 2003.

7. Röösli M, Egger M, Pfluger D and Minder C: Cardiovascular mortality and exposure to extremely low frequency magnetic fields: A cohort study of Swiss railway workers. Environ Health 7: 35, 2008.

8. Genuis SJ and Lipp CT: Electromagnetic hypersensitivity: Fact or fiction? Sci Total Environ 414: 103-112, 2012.

9. Ahlbom A, Day N, Feychting M, Roman E, Skinner J, Dockerty J, Linet M, McBride M, Michaelis J, Olsen JH, et al: A pooled analysis of magnetic fields and childhood leukaemia. Br J Cancer 83 692-698, 2000.

10. Greenland S, Sheppard AR, Kaune WT, Poole C and Kelsh MA: A pooled analysis of magnetic fields, wire codes, and childhood leukemia. Childhood Leukemia-EMF Study Group. Epidemiology 11: 624-634, 2000.

11. Kheifets L, Afifi AA and Shimkhada R: Public health impact of extremely low-frequency electromagnetic fields. Environ Health Perspect 114: 1532-1537, 2006.

12. Kheifets L, Ahlbom A, Crespi CM, Draper G, Hagihara J, Lowenthal RM, Mezei G, Oksuzyan S, Schüz J, Swanson J, et al: Pooled analysis of recent studies on magnetic fields and childhood leukaemia. Br J Cancer 103: 1128-1135, 2010.
13. Hardell L and Sage C: Biological effects from electromagnetic field exposure and public exposure standards. Biomed Pharmacother 62: 104-109, 2008.

14. Balkwill F: Cancer and the chemokine network. Nat Rev Cancer 4: 540-550, 2004.

15. Ishida $\mathrm{T}$, Utsunomiya A, Iida $\mathrm{S}$, Inagaki $\mathrm{H}$, Takatsuka $\mathrm{Y}$, Kusumoto S, Takeuchi G, Shimizu S, Ito M, Komatsu H, et al: Clinical significance of CCR4 expression in adult T-cell leukemia/lymphoma: Its close association with skin involvement and unfavorable outcome. Clin Cancer Res 9: 3625-3634, 2003.

16. Itatani $\mathrm{Y}$, Kawada $\mathrm{K}$, Inamoto S, Yamamoto $\mathrm{T}$, Ogawa $\mathrm{R}$, Taketo MM and Sakai Y: The role of chemokines in promoting colorectal cancer invasion/metastasis. Int J Mol Sci 17: 643, 2016.

17. Kitamura T, Qian BZ, Soong D, Cassetta L, Noy R, Sugano G, Kato Y, Li J and Pollard JW: CCL2-induced chemokine cascade promotes breast cancer metastasis by enhancing retention of metastasis-associated macrophages. J Exp Med 212: 1043-1059, 2015.

18. Roy I, McAllister DM, Gorse E, Dixon K, Piper CT, Zimmerman NP, Getschman AE, Tsai S, Engle DD, Evans DB, et al: Pancreatic cancer cell migration and metastasis is regulated by chemokine-biased agonism and bioenergetic signaling. Cancer Res 75: 3529-3542, 2015.

19. Struyf S, Schutyser E, Gouwy M, Gijsbers K, Proost P, Benoit Y, Opdenakker G, Van Damme J and Laureys G: PARC/CCL18 is a plasma CC chemokine with increased levels in childhood acute lymphoblastic leukemia. Am J Pathol 163: 2065-2075, 2003.

20. Till KJ, Lin K, Zuzel M and Cawley JC: The chemokine receptor CCR7 and alpha4 integrin are important for migration of chronic lymphocytic leukemia cells into lymph nodes. Blood 99: 2977-2984, 2002.

21. Schröttner P, Wollner S, Catusse J and Burger M: Detection of elevated serum levels of the chemokine CCL18 in B-cell chronic lymphocytic leukaemia: Identification of a novel biomarker. Acta Haematol 124: 110-114, 2010.

22. Lynch HE, Sanchez AM, D'Souza MP, Rountree W, Denny TN, Kalos $\mathrm{M}$ and Sempowski GD: Development and implementation of a proficiency testing program for Luminex bead-based cytokine assays. J Immunol Methods 409: 62-71, 2014.

23. Cardona AE, Sasse ME, Liu L, Cardona SM, Mizutani M, Savarin C, Hu T and Ransohoff RM: Scavenging roles of chemokine receptors: Chemokine receptor deficiency is associated with increased levels of ligand in circulation and tissues. Blood 112: 256-263, 2008

24. Li L, Xiong DF, Liu JW, Li ZX, Zeng GC and Li HL: No effects of power line frequency extremely low frequency electromagnetic field exposure on selected neurobehavior tests of workers inspecting transformers and distribution line stations versus controls. Australas Phys Eng Sci Med 37: 37-44, 2014.

25. He S, He S, Chen CH, Deborde S, Bakst RL, Chernichenko N, McNamara WF, Lee SY, Barajas F, Yu Z, et al: The chemokine (CCL2-CCR2) signaling axis mediates perineural invasion. Mol Cancer Res 13: 380-390, 2015.

26. Zsiros E, Duttagupta P, Dangaj D, Li H, Frank R, Garrabrant T, Hagemann IS, Levine BL, June CH, Zhang L, et al: The ovarian cancer chemokine landscape is conducive to homing of vaccine-primed and CD3/CD28 costimulated T cells prepared for adoptive therapy. Clin Cancer Res 21: 2840-2850, 2015.

27. Brown CE, Vishwanath RP, Aguilar B, Starr R, Najbauer J, Aboody KS and Jensen MC: Tumor-derived chemokine MCP-1/CCL2 is sufficient for mediating tumor tropism of adoptively transferred T cells. J Immunol 179: 3332-3341, 2007.

28. Lu Y, Chen Q, Corey E, Xie W, Fan J, Mizokami A and Zhang J: Activation of MCP-1/CCR2 axis promotes prostate cancer growth in bone. Clin Exp Metastasis 26: 161-169, 2009.

29. Conti P and DiGioacchino M: MCP-1 and RANTES are mediators of acute and chronic inflammation. Allerg Asthm Proc 22: 133-137, 2001.

30. Fujimoto H, Sangai T, Ishii G, Ikehara A, Nagashima T, Miyazaki M and Ochiai A: Stromal MCP-1 in mammary tumors induces tumor-associated macrophage infiltration and contributes to tumor progression. Int J Cancer 125: 1276-1284, 2009

31. Reale M, De Lutiis MA, Patruno A, Speranza L, Felaco M, Grilli A, Macrì MA, Comani $\mathrm{S}$, Conti $\mathrm{P}$ and Di Luzio $\mathrm{S}$ : Modulation of MCP-1 and iNOS by $50-\mathrm{Hz}$ sinusoidal electromagnetic field. Nitric Oxide 15: 50-57, 2006. 
32. D'Angelo C, Costantini E, Kamal MA and Reale M: Experimental model for ELF-EMF exposure: Concern for human health. Saudi J Biol Sci 22: 75-84, 2015.

33. Vianale G, Reale M, Amerio P, Stefanachi M, Di Luzio S and Muraro R: Extremely low frequency electromagnetic field enhances human keratinocyte cell growth and decreases proinflammatory chemokine production. Br J Dermatol 158: $1189-1196,2008$

34. Levina V, Nolen BM, Marrangoni AM, Cheng P, Marks JR, Szczepanski MJ, Szajnik ME, Gorelik E and Lokshin AE: Role of eotaxin-1 signaling in ovarian cancer. Clin Cancer Res 15: 2647-2656, 2009.

35. Salcedo R, Young HA, Ponce ML, Ward JM, Kleinman HK, Murphy WJ and Oppenheim JJ: Eotaxin (CCL11) induces in vivo angiogenic responses by human CCR3+ endothelial cells J Immunol 166: 7571-7578, 2001.
36. Zhu F, Liu P, Li J and Zhang Y: Eotaxin-1 promotes prostate cancer cell invasion via activation of the CCR3-ERK pathway and upregulation of MMP-3 expression. Oncol Rep 31: 2049-2054, 2014.

37. Koç Ü, Çetinkaya E, Bostanci EB, Kemık AS, Tez M, Gömceli I and Akoğlu M: Diagnostic significance of serum eotaxin-1 level in gastric cancer patients. Dis Markers 35: 363-367, 2013.

38. Nguyen AH, Lee J, Il Choi H, Seok Kwak H and Jun Sim S: Fabrication of plasmon length-based surface enhanced Raman scattering for multiplex detection on microfluidic device. Biosens Bioelectron 70: 358-365, 2015.

(i)(9) This work is licensed under a Creative Commons Attribution-NonCommercial-NoDerivatives 4.0 International (CC BY-NC-ND 4.0) License. 